\title{
Hepatic epithelioid haemangioendothelioma: difficult name, difficult diagnosis?
}

\author{
I. Cobden, ${ }^{1}$ S. Johri, ${ }^{1}$ G. Terry, ${ }^{2}$ K.B. Robinson, ${ }^{2}$ M.K. Bennett ${ }^{3}$ and \\ R. Lendrum ${ }^{3}$ \\ ${ }^{1}$ Preston Hospital, North Tyneside, NE29 0LR, ${ }^{2}$ Dryburn Hospital, Durham, DH1 5TW and ${ }^{3}$ Freeman \\ Hospital, Newcastle upon Tyne, NE7 7DN, UK.
}

\begin{abstract}
Summary: Two patients with epithelioid haemangioendotheliomata of the liver are described. Both presented with abdominal pain and malaise, with hepatomegaly and a variable degree of hepatocellular dysfunction. Diagnosis was delayed in both cases, each patient undergoing a protracted series of investigations including repeated liver biopsies. The major obstacles to early diagnosis were a lack of clinical awareness of the condition and difficulties in interpretation of the liver histology: the widespread sclerosis in the tumour tissue is easily mistaken for a post-necrotic or cirrhotic process. The key to the diagnosis is the demonstration of cells containing Factor-VIIIrelated antigen confirming the endothelial origin of the tumour. One patient died within three months of presentation and the other after 18 months. The tumour may, therefore, be more aggressive than earlier reports seem to suggest. It seems likely that the tumour is being under-diagnosed and although no specific therapy has been shown to be of value, a greater awareness of the condition, resulting in a more prompt diagnosis, should save patients from undergoing unnecessary investigation.
\end{abstract}

\section{Introduction}

Epithelioid haemangioendotheliomata comprise a comparatively 'new' group of tumours. The first description of such tumour in lung was probably that of the 'intravascular bronchioloalveolar tumour' described in $1975,{ }^{1}$ although there were earlier reports which probably refer to the same condition over the previous decade. Its vascular origin was not recognised until $1979,{ }^{2}$ since when there have been a number of reports of the tumour, mostly related to blood vessels in the extremities. ${ }^{3}$ A variety of names including 'sclerosing endothelial tumour' and 'sclerosing epithelioid angiosarcoma' have been applied to this tumour. Some reports mention liver involvement, usually considering this to be metastatic, but in 1984 a series of 32 primary hepatic haemangioendotheliomata, gleaned from a survey of 17 years of hepatic histology was

Correspondence: I. Cobden M.D., M.R.C.P.

Accepted: 23 September 1987 described $^{4}$ and in 1985 Dean and colleagues 5 reviewed five patients, all young women with a history of oral contraceptive use. The implication that hepatic tumours may not be excessively rare is supported by the following case reports of two patients who presented within a year to neighbouring district hospitals.

\section{Case reports}

\section{Patient 1}

A 53 year old woman presented with a one year history of fatigue, weight loss and right upper abdominal discomfort. She had always been in good health beforehand. Physical examination was normal except for striking hepatomegaly. The serum alkaline phosphatase was markedly elevated. An isotope liver scan showed hepatomegaly with multiple filling defects present; moderate spleno-

(C) The Fellowship of Postgraduate Medicine 1988 
megaly was shown. Ultrasonography showed an irregular echo pattern in an enlarged liver. Liver biopsy was reported as showing some fatty degeneration of liver cells but nothing else of note. Further laparoscopic biopsy was undertaken and reported as showing areas of coarse portal tract fibrosis and marked fatty change suggesting alcohol excess with no evidence of cirrhosis or malignancy.

Eight months after her initial presentation her clinical condition began to deteriorate with progressive ascites. Blood tests included bilirubin $20 \mu \mathrm{mol} / \mathrm{l}$, alkaline phosphatase $1453 \mathrm{IU} / 1$ (normal $<300$ ) aspartate amino transferase (AST) $43 \mathrm{IU} / \mathrm{l}$, gamma glutamyl transpeptidase $188 \mathrm{IU} / \mathrm{l}$, albumin $31 \mathrm{~g} / \mathrm{l}, \alpha$-fetoprotein levels not elevated, and positive hepatitis B surface and core antibodies. She was then transferred to the gastroenterology unit for further investigation by which time she was clinically jaundiced, with palmar erythema, spider naevi and asterixis. A hard liver edge was palpable $8 \mathrm{~cm}$ below the costal margin. There was tense ascites which slowly responded to diuretics. Endoscopic retrograde cholangiopancreatography (ERCP) revealed a markedly compressed common bile duct and common hepatic duct; calcification was also present in the liver parenchyma. A computed tomographic (CT) scan (Figure 1) confirmed an enlarged liver of mainly low attenuation perhaps representing areas of regeneration within a largely necrotic liver. A further liver biopsy was thought to be compatible with this CT diagnosis but this did not seem consistent with the gross hepatomegaly, however, and the slides were referred to further expert pathologists. It was revealed for the first time that the liver contained an unusual neoplasm; the diagnosis was subsequently confirmed by positive immunocytochemistry for factor VIII within the tumour.

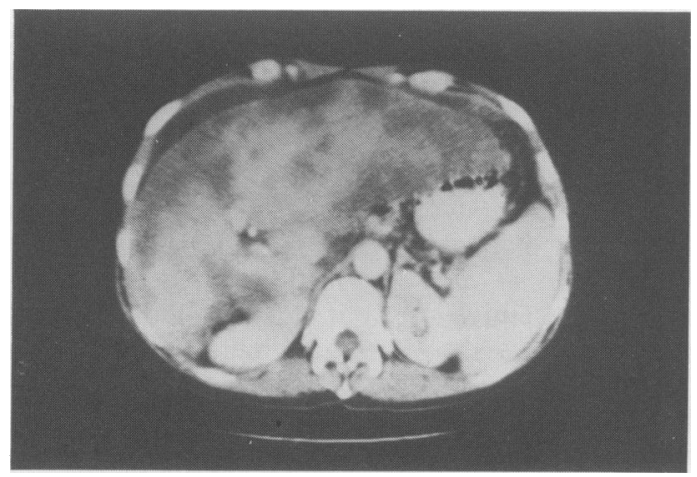

Figure 1 CT scan of upper abdomen showing grossly enlarged liver of low attenuation with defects in both lobes.
Over the following eight months she required repeated admission with signs of progressive hepatic insufficiency and eventually died 18 months after initial presentation. Post-mortem examination revealed a liver weighing $2100 \mathrm{~g}$; the right and left lobes appeared to be replaced by white fibrous tissue (Figure 2). There was no invasion of hepatic veins but oesophageal varices were present and the spleen weighed $310 \mathrm{~g}$. Tumour had invaded the right hemidiaphragm and one small subpleural metastasis was detected. Histology (Figures 3 and 4) of the tumour tissue shows the typical appearances at low and high power.

\section{Patient 2}

A 64 year old woman presented with a three month history of nausea, vomiting, anorexia and weight loss of two stones. She had had a successful closed mitral valvotomy 16 years previously and stable angina of effort for four years. Current treatment consisted of nifedipine and digoxin. On examination she was thin, but not pale or icteric. She was in controlled atrial fibrillation with signs of mild mitral stenosis. Abdominal examination was unremarkable and a full blood count was normal. The initial clinical diagnosis of a gastric ulcer or carcinoma was excluded by upper gastrointestinal endoscopy. Liver function tests were markedly abnormal, however, with an alkaline phosphatase of $406 \mathrm{IU} / 1$ (normal <93) and $\gamma$-glutamyl transpeptidase of $211 \mathrm{IU} / 1$ (normal <65). Bilirubin, transaminases and protein levels were within normal limits. Serum markers for hepatitis B and an auto-antibody screen were negative. Upper abdominal ultrasonography was non-contributory, but a technetium-sulphur colloid isotope scan confirmed enlargement of the left lobe of the liver

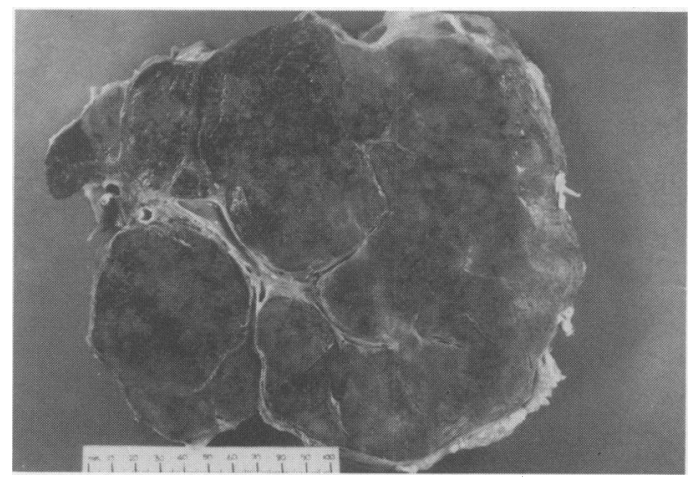

Figure 2 Cut surface of liver at post-mortem showing most of the left lobe and part of the right lobe replaced by 'fibrous' tissue. 


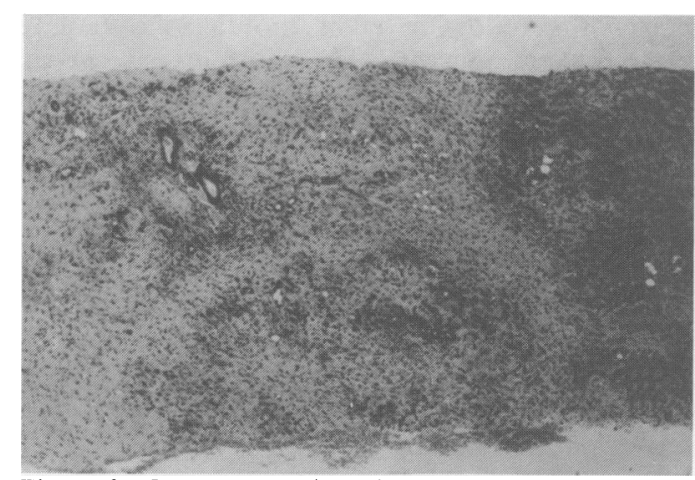

Figure 3 Low power view showing infiltration of the lobule and portal tract with residual bile ducts surrounded by a bland, but cellular infiltrate. The tumour extends between sinusoids and bile ducts (original magnification $\times 51$ ).

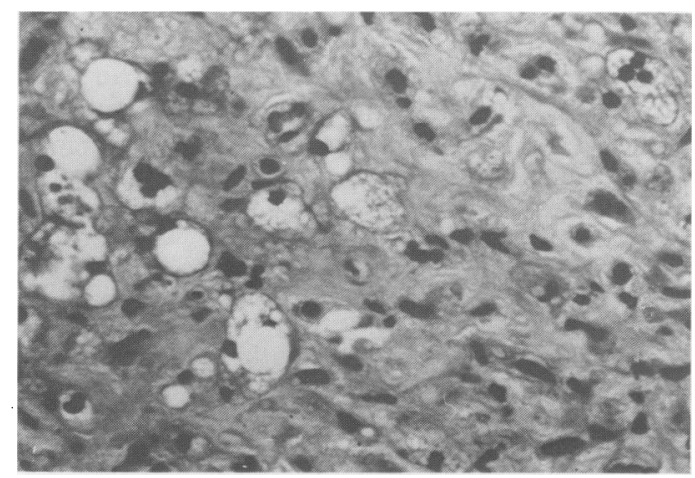

Figure 4 High power view shows typical epithelioid cells with a slightly vacuolated cytoplasm admixed with the spindle cells. No obvious vascular channels are recognised. (H\&E original magnification $\times 320$ ).

with filling defects in the right lobe. A percutaneous needle biopsy of liver produced a core of white tissue, reported on histology to consist of fibrous tissue with no hepatic structures evident apart from a few hepatocytes in a rather disorganised pattern at one end of the specimen. A repeat liver biopsy was performed (Figure 5), two strips of tissue being obtained, one reported as being almost completely replaced by fibrosis and the other showing nodules of normal tissue with fatty change surrounded by fibrosis, interpreted as a cirrhosis with no definite evidence of malignancy. By this stage she had developed a low grade pyrexia and a neutrophil leucocytosis, so to exclude a co-existing choledocholithiasis (notwithstanding the normal ultrasound report) endoscopic cholangiography was performed. This demonstrated some abnormalities of intrahepatic radicals, but no extrahepatic biliary

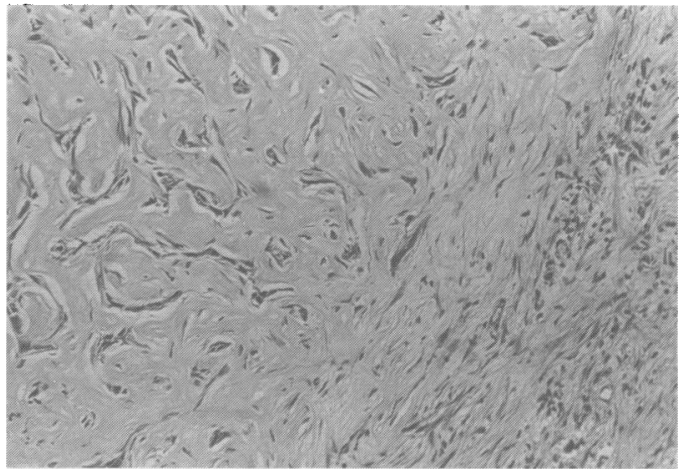

Figure 5 Most of the biopsy showed extensive collagen deposition with strands of mildly pleomorphic cells irregularly arranged. Most of these cells are spindle-shaped with occasional mitoses present. (H\&E, original magnification $\times 205$ ).

obstruction. However, as a result of discussions at this shared ERCP list, when one of the authors (RL) noted the similarities to the previous patient, it was decided to review the sections including staining for Factor VIII-related-antigen. The diagnosis of epithelioid haemangioendothelioma was confirmed, the report arriving on the day after the patient died suddenly at home. Post-mortem examination was not carried out.

\section{Discussion}

The clinical presentation and pathology of the tumour in these patients correspond very closely to the detailed descriptions provided by Ishak et al. ${ }^{4}$ In their series most patients presented with vague malaise and abdominal discomfort, although in a fifth the tumour appeared to be an incidental finding. Two-thirds of patients had an elevated serum alkaline phosphatase which was the major biochemical abnormality in our patients. In threequarters of them the tumour nodules were multiple and calcification (as seen in our first patient) was detected in about $20 \%$ radiologically and about $30 \%$ histologically. Staining for Factor-VIII-related antigen is the key to the diagnosis, although the proportion of reacting cells may be less than onethird. Dean et $a l^{5}{ }^{5}$ described an association with oral contraceptive use, but neither of our patients had been exposed to hormonal therapy of any kind.

These two case reports highlight the diagnostic difficulties posed by this tumour. The two patients underwent a total of five liver biopsies (not to mention numerous other procedures and investigations) without the diagnosis or even the neoplastic nature of the condition, being suspected. 
The cases therefore emphasize the importance of seeking further expert opinion about difficult hepatic histology. Without this the first patient possibly and the second almost certainly would have died undiagnosed.

If our experience is mirrored elsewhere then the tumour is almost certainly being missed and may be more common than hitherto recognised. The extensive sclerosis superficially resembles a cirrhosis or post-massive necrosis, ${ }^{4}$ although it is clear that neither the pathologists nor clinicians involved were completely happy with these diagnoses in our cases. We suspect that a number of patients with hepatic epithelioid haemangioendothelioma may be dying with the label 'cryptogenic cirrhosis' or possibly, if no histology is obtained, of 'metastatic tumour, unknown primary'. Other mis-diagnoses include veno-occlusive disease, although the invasion of portal and hepatic veins and abundant fibrous tissue are a clue to the malignant potential. Even if the neoplastic nature is recognized the tumour may be considered to be of epithelial origin, ${ }^{3}$ perhaps a hepatocellular or cholangiocarcinoma ${ }^{4}$ which can occasionally manifest considerable sclerosis.

\section{References}

1. Dail, D.H. \& Liebow, A.A. Intravascular bronchioloalveolar tumour. Am J Pathol 1975, 78: 61a.

2. Corrin, B., Manners, B., Millard, M. \& Weaver, L. Histogenesis of the so-called intravascular bronchioloalveolar tumour'. J Pathol 1979, 128: 163-167.

3. Weiss, S.W. \& Enzinger, F.M. Epithelioid haemangioendothelioma: a vascular tumour often mistaken for a carcinoma. Cancer 1982, 50: 970-981.

4. Ishak, K.G., Sesterhenn, I.A., Goodman, Z.D., Rabin, L. \& Stromeyer, F.W. Epithelioid haemangioendothelioma of the liver: a clinicopathologic and follow-up study of 32 cases. Hum Pathol 1984, 15: 839-852.
Finally, the prognosis for the tumour, sometimes regarded as benign, must be guarded. Although in Ishak's retrospective series three of their 32 patients died within two years of diagnosis, many were alive for at least five years with the longest survivor dying 28 years after discovery of the tumour. For reasons mentioned above, however, it may be that patients with more aggressive tumours are more likely to die without the correct diagnosis being made. Apart from one report of resection, ${ }^{5}$ treatment appears to make little difference.

In conclusion we hope that by drawing attention to this tumour it may be possible to avoid further patients being subjected to a protracted series of unnecessary, uncomfortable and expensive investigations.

\section{Acknowledgements}

We wish to thank Professor P.J. Scheuer and Professor R. McSween for their expert opinion. Mrs L. Gittins prepared the manuscript.

5. Dean, P.J., Haggit, R.C. \& O'Hara, C.J. Malignant epithelioid haemangioendothelioma of the liver in young women: relationship to oral contraceptive use. Am J Surg Pathol 1985, 9: 695-704. 